\title{
Conservation between higher plants and the moss Physcomitrella patens in response to the phytohormone abscisic acid: a proteomics analysis
}

Xiaoqin Wang ${ }^{1,2,3,4}$, Tingyun Kuang ${ }^{1}$, Yikun $\mathrm{He}^{1 *}$

\begin{abstract}
Background: The plant hormone abscisic acid ( $A B A)$ is ubiquitous among land plants where it plays an important role in plant growth and development. In seeds, ABA induces embryogenesis and seed maturation as well as seed dormancy and germination. In vegetative tissues, $A B A$ is a necessary mediator in the triggering of many of the physiological and molecular adaptive responses of the plant to adverse environmental conditions, such as desiccation, salt and cold.
\end{abstract}

Results: In this study, we investigated the influence of abscisic acid (ABA) on Physcomitrella patens at the level of the proteome using two-dimensional gel electrophoresis (2-DE) and liquid chromatography-tandem mass spectrometry (LC-MS/MS). Sixty-five protein spots showed changes in response to ABA treatment. Among them, thirteen protein spots were down-regulated; fifty-two protein spots were up-regulated including four protein spots which were newly induced. These proteins were involved in various functions, including material and energy metabolism, defense, protein destination and storage, transcription, signal transduction, cell growth/division, transport, and cytoskeleton. Specifically, most of the up-regulated proteins functioned as molecular chaperones, transcriptional regulators, and defense proteins. Detailed analysis of these up-regulated proteins showed that ABA could trigger stress and defense responses and protect plants from oxidative damage. Otherwise, three protein kinases involved in signal pathways were up-regulated suggesting that $P$. patens is sensitive to exogenous ABA. The down-regulated of the Rubisco small subunit, photosystem II oxygen-evolving complex proteins and photosystem assembly protein ycf3 indicated that photosynthesis of P. patens was inhibited by ABA treatment.

Conclusion: Proteome analysis techniques have been applied as a direct, effective, and reliable tool in differential protein expressions. Sixty-five protein spots showed differences in accumulation levels as a result of treatment with ABA. Detailed analysis these protein functions showed that physiological and molecular responses to the plant hormone ABA appear to be conserved among higher plant species and bryophytes.

\section{Background}

Plants undergo continuous exposure to various biotic and abiotic stresses in their natural environment. To survive under such conditions, plants activate signaling cascades that lead to the accumulation of endogenous hormones which, in turn, trigger the induction of defense responses. Abscisic acid (ABA) is a small,

\footnotetext{
* Correspondence: yhe@mail.cnu.edu.cn

${ }^{1}$ College of Life Sciences, Capital Normal University, Beijing 100048, China Full list of author information is available at the end of the article
}

lipophilic phytohormone that regulates many important aspects of plant growth and development and plays a crucial role in cellular responses to environmental stresses such as drought, cold, salt, wounding, UV radiation, and pathogen attack [1]. It is ubiquitous in lower and higher plants and has also been found in algae [2], fungi [3], and even mammalian brain tissue [4].

Many studies have been carried out that characterize ABA signaling pathways and physiological responses to ABA in higher plants. Mutational analyses in Arabidopsis have led to the identification of several genes that

() 
are involved in ABA signaling pathways. The proteins involved in ABA signaling pathways include $G$ proteins, protein phosphatases, and protein kinases [5]. Target proteins that respond to ABA can be directly involved in cellular protection or can act as transcriptional factors that link extracellular signaling to the regulation of transcription in eukaryotic cells an important part of the physiological response to ABA is achieved through gene expression and protein synthesis. Transcriptome analyses have shown that ABA dramatically alters gene expression. More than 1300 ABA-regulated genes were identified by random mass sequencing of Arabidopsis transcripts [6,7]. Proteome research has also indicated that exogenous ABA can induce the synthesis of many proteins in rice seedlings. These proteins are involved in signaling pathways, transcription, cell growth and division, photosynthesis, protein synthesis and trafficking, and defense/stress-response, among others [8]. To date however, relatively little is known regarding ABA signaling pathways in bryophytes.

Bryophytes were the first land plants and they have been a powerful experimental tool and model for the elucidation of complex biological processes [9-11]. Evolutionary studies have indicated that bryophytes may form a sister clade with tracheophytes (vascular plants). Analysis of organisms from these ancient clades can make significant contributions to understanding the development [12], physiology [13], phylogenetics [14] and stress-induced cellular responses among plants [15]. Recently, comparison of the draft genome sequence of $P$. patens with the genomes of flowering plants revealed evolutionary insights into the conquest of land by plants. Furthermore, bryophyte physiology is regulated by many of the same phytohormones used by higher plants (e.g., auxins, abscisic acid, and gibberellins) and uses similar mechanisms of intracellular messaging (e.g. Ca2+) found in higher plants $[13,16,17]$.

In recent years, the moss $P$. patens become a new model for studying the phytohormone ABA. ABA is involved in abiotic stress adaptation of $P$. patens, since exogenously applied ABA increases freezing tolerance [18] and increased endogenous ABA levels are detected upon osmotic stress treatment [19]. Moreover, plants like $P$. patens comprising both protonemata and gametophores will tolerate complete desiccation following slow drying, if first pretreated with $\mathrm{ABA}$, a process that is associated with a substantial increase in intracellular levels of sucrose [20] as is cold acclimation leading to freezing tolerance [21]. ABA-induced stress tolerance in $P$. patens is also accompanied by increased expression of stress-related genes [6,7]. However, the role of ABA or the signaling pathways in response to environmental stresses in the moss $P$. patens has yet to be elucidated.
Here, proteomics has been used to increase understanding of ABA signaling pathways and defense responses in $P$. patens. We have examined the global changes of proteins in $P$. patens following ABA treatment using two dimensional gel electrophoresis (2-DE) coupled with LC-MS/MS. Sixty-five proteins have been identified as being up- or down-regulated in response to ABA treatment. These proteins are involved in metabolism and energy, defense responses, protein trafficking and storage, transcription, and signaling pathways, as well as others. Our study reveals the complex signaling pathways and defense responses by which ABA functions in $P$. patens. It also suggests that molecular responses to ABA may be highly conserved between higher plant species and bryophytes.

\section{Results}

\section{Proteome profile changes in $P$. patens under ABA} treatment

Changes in the proteome profile between control and ABA treated plants suggested that exogenous ABA could trigger one or more responses in $P$. patens. In order to ensure statistically results, the experiments were carried out in triplicate. After CBB R-250 staining, more than 1,300 protein spots could be detected for each sample. Representative gels from control and ABAtreated plants and proteins showing altered abundance are presented in Figure 1. Two-dimensional images were analyzed using ImageMaster ${ }^{\mathrm{TM}} 2 \mathrm{D}$ Platinum software. The volume percentage of each spot was estimated and compared across the gels. In the samples following ABA treatment, we repeatedly analyzed 89 protein spots, whose relative abundance was at least 1.5 -fold different from the control. The proteins associated with 65 of these protein spots were subsequently identified using LC-MS/MS. Among them, 13 protein spots (D1-D13) were down-regulated, 52 protein spots (U1-U52) were up-regulated by ABA treatment including four protein spots (U22, U24, U40, U43) which were only present in the ABA-treated samples (Figure 1). Quantitative analysis indicated that the abundance of these proteins changed [see Additional File 1: Supplemental Table S1] in response to ABA treatment (Figure 2) suggesting that different physiological and biochemical processes are modified following ABA treatment.

\section{Protein identification by LC-MS/MS}

The protein spots of interest were excised from preparative 2-D gels. After trypsin autolysis, the digested spots were analyzed by LC-MS/MS. The resulting amino acid sequences were searched against databases using SEQEST software. As the $P$. patens sequence information is limited, there were some samples that did not yield 


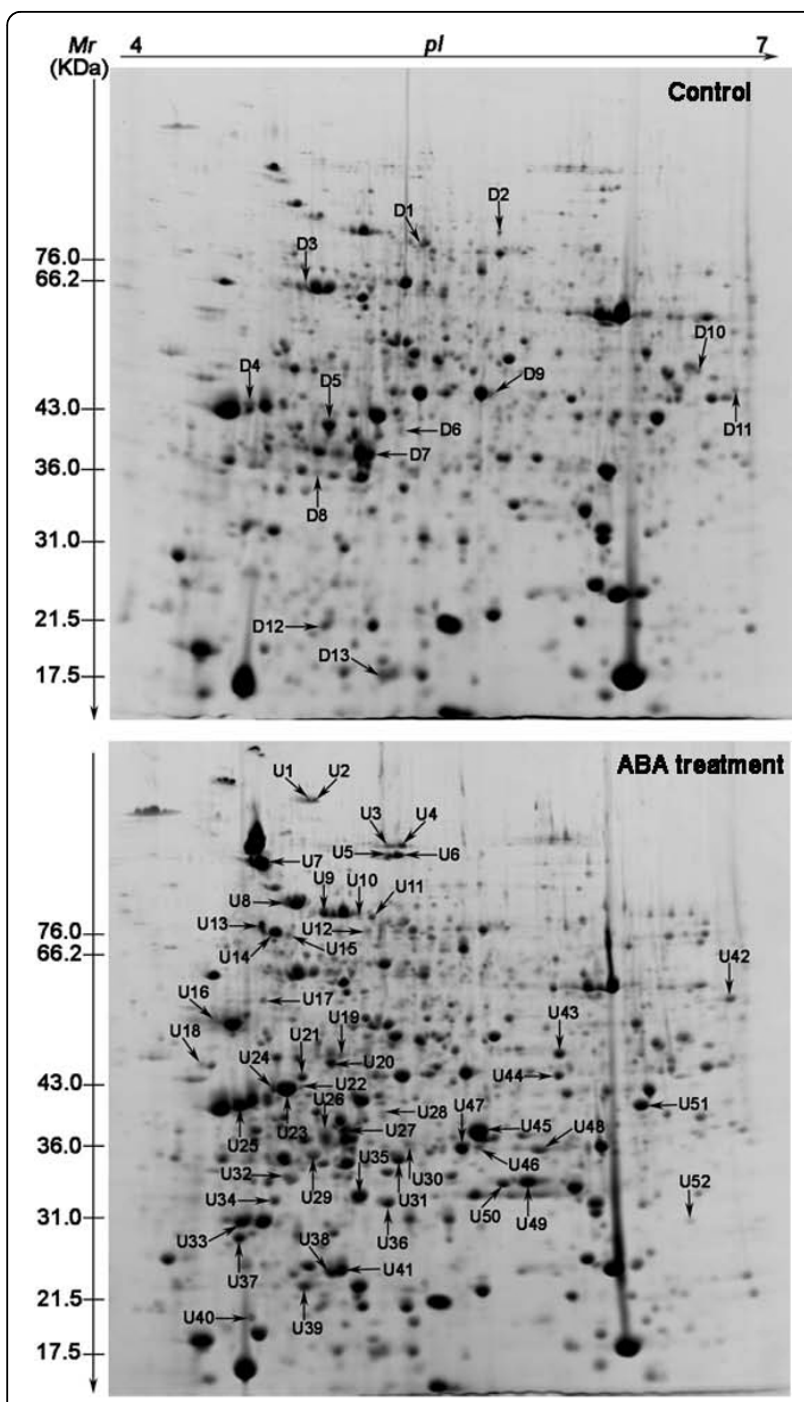

Figure 1 Comparison of the proteome pattern of $P$. patens gamatophores under control and ABA treatments. The arrows indicate the ABA-responsive proteins. Down-regulated proteins (spots D1-D13) are indicated in the control image. Up-regulated proteins and newly-induced proteins (spots U1-U52) are indicated in the image following $50 \mu \mathrm{M}$ ABA treatment.

significant matches. For analysis of these samples, the Arabidopsis database was used. Previous transcriptome research has shown that the gene set in $P$. patens is very similar to that of $A$. thaliana [22]. The results of the database search are listed in Table 1 and Supplemental Table S2 [see Additional File 2: Supplemental Table S2].

\section{Functional categorization of $A B A$-responsive proteins}

The ABA-responsive proteins could be sorted into eight categories (Figure 3, Table 2) according to their biological function as described by the EU A. thaliana genome project [23]. These categories include material and energy metabolism $(28.9 \%)$, defense $(26.7 \%)$, protein synthesis and degradation (17.8\%), transcription (11.1\%), signal transduction (6.7\%), cell growth/division (4.4\%), transport (2.2\%), and cytoskeleton (2.2\%).

Within the material and energy metabolism category, ATP synthase beta subunit (spot D3), putative protein 1 photosystem II oxygen-evolving complex (spot D4), rubisco small subunit (spots D9, D13) and photosystem assembly protein ycf3 (spot D12) were down-regulated, and another nine proteins were up-regulated. All proteins within the defense category were up-regulated. These proteins include putative proline-rich protein (spot U15), putative expansin (spot U18), extensin-like protein (spot U19), intracellular pathogenesis-related protein-like protein (spot U24), disease resistance protein RPM1 (spot U29), lipoxygenase (spot U30), thylakoid-bound ascorbate peroxidase (spot U33), 2-Cys peroxiredoxin (spot U37), physcomitrin (spots U38, U41), N-hydroxycinnamoyl/benzoyltransferase-like protein (spot U42), putative ascorbate peroxidase (spots U45, U46) and peroxiredoxin (spot U50). Proteins involved in protein synthesis, degradation and folding include different family members of the HSP70 family (spots U7, U8, U9, U10, U11, U25, U35 and U52), chloroplast ftsH protease (spot D1) and cullin 3a (spot U27). The up-regulated proteins probable Myb-family transcription factor (spot U2), fibrillarin-like protein (spot U16), probable WRKY transcription factor 52 (spot U22), bZIP transcription factor-like protein (spot U26) and mRNA binding protein precursor-like (spot U44) are all related to transcription. Some signal pathway proteins were also up-regulated, including calciumdependent protein kinase-like protein (spot U4), ser/thr specific protein kinase-like protein (spot U5) and putative receptor-like protein kinase (spot U14).

\section{Discussion}

In seed plants, the phytohormone ABA controls many developmental and physiological processes via complicated signaling networks that are composed of receptors, secondary messengers, protein kinase, and transcription factors. Transcription factors can meditate protein synthesis in responses to ABA, which is crucial in plant growth and development as well as in plant responses to various stresses. In this study, a broad spectrum of proteins is synthesized following ABA treatment. Detailed analysis of these proteins indicates that the molecular responses to the plant hormone ABA appears to be conserved among higher plant species and bryophytes, as discussed below.

\section{ABA induced signaling proteins in $P$. patens}

Receptor protein kinases (spot U14) are plasma membrane-bound and play an important role in the perception and transmittance of external signals such as ABA, 


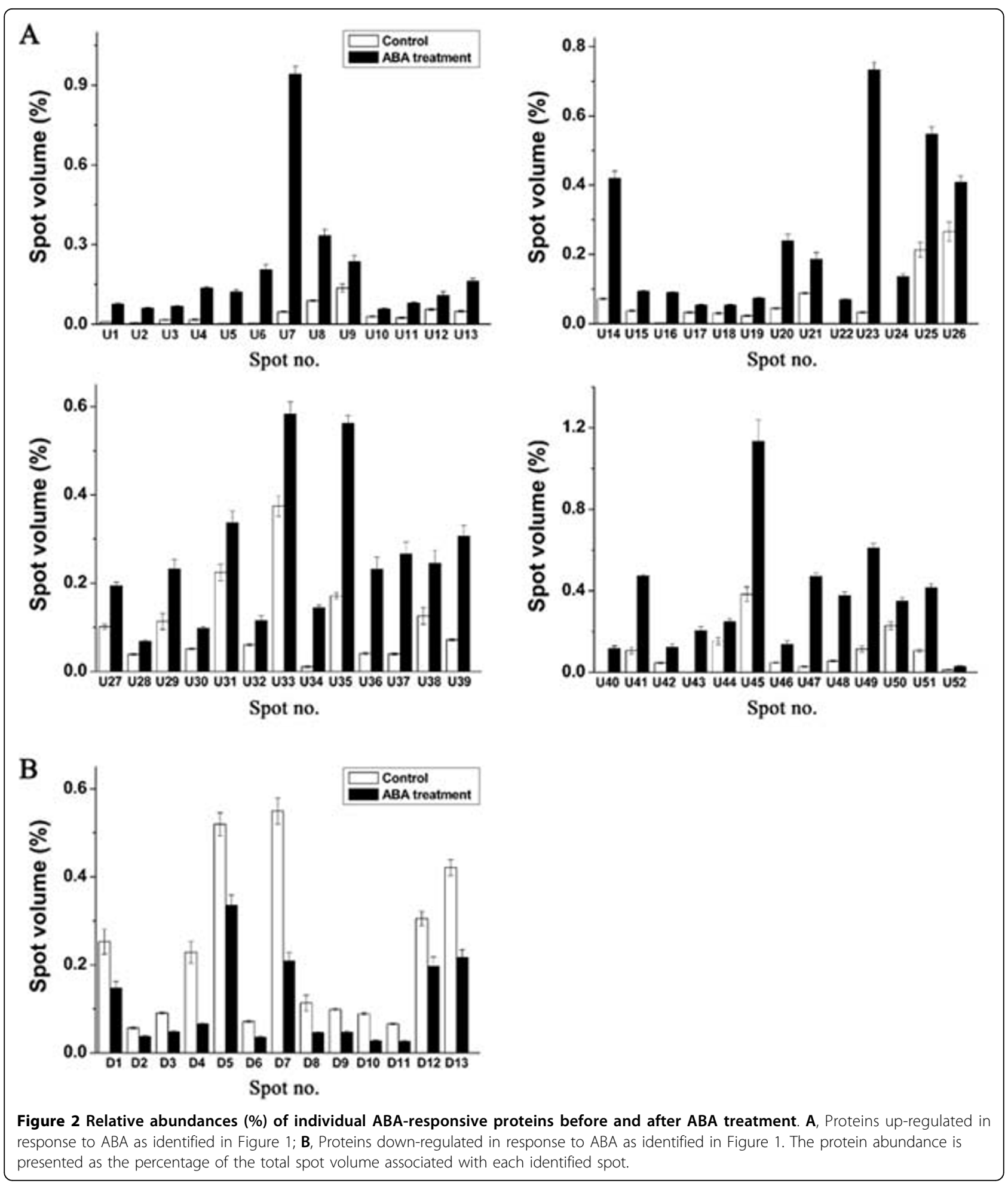

dehydration, high salt and cold treatments [24-26]. Calcium-dependent protein kinases (CDPKs) (spot U4) are implicated as important sensors of $\mathrm{Ca}^{2+}$ flux in plants in response to a variety of environmental stimuli and hormone signals. Depending upon the calcium signature, the extent and duration of CDPK enzyme activation will vary, but will have a direct effect on the phosphorylation status of its downstream targets [27]. Ser/Thr specific protein kinase (spot U5) catalyzes ATP-dependent phosphorylation of serine and threonine residues on target 
Table 1 Identification of ABA-responsive proteins in $P$. patens

\begin{tabular}{|c|c|c|c|c|c|c|c|}
\hline $\begin{array}{l}\text { Spot } \\
\text { no. }\end{array}$ & Accession no. & $\begin{array}{l}\text { Theor./exp. } \\
\text { Mass (kDa) }\end{array}$ & Theor./exp. pl & Description & NMP & SC (\%) & Species \\
\hline D1 & CAA68141.1 & $76.1 / 77.8$ & $5.83 / 5.44$ & Chloroplast FtsH protease & 6 & 16.22 & A. thaliana \\
\hline D2 & CAB79006.1 & $127.3 / 81.2$ & $7.92 / 5.81$ & Hypothetical protein & 1 & 1.59 & A. thaliana \\
\hline D3 & BAC85045.1 & $53.3 / 68.3$ & $4.98 / 4.88$ & ATP synthase beta subunit & 18 & 70.04 & P. patens \\
\hline D4 & CAB42911.1 & $35.0 / 45.7$ & $5.92 / 4.61$ & Putative protein 1 photosystem II oxygen-evolving complex & 2 & 8.76 & A. thaliana \\
\hline D5 & E86427 & $57.4 / 42.6$ & 7.63/4.99 & Hypothetical protein T4k22.7 & 1 & 2.95 & A. thaliana \\
\hline D6 & P46875 & $85.0 / 41.6$ & $5.9 / 5.35$ & Kinesin-3(Kinesin-like protein C) & 1 & 1.72 & A. thaliana \\
\hline D7 & CAC43717.1 & $49.7 / 38.4$ & $6.09 / 5.19$ & Unnamed protein product & 2 & 6.74 & P. patens \\
\hline D8 & CAC43713.1 & $31.9 / 36.1$ & $5.36 / 4.94$ & Unnamed protein product & 10 & 40.98 & P. patens \\
\hline D9 & BAC87878.1 & $21.1 / 47.2$ & $8.77 / 5.77$ & Rubisco small subunit & 5 & 45.65 & P. patens \\
\hline D10 & CAB80784.1 & $55.7 / 50.9$ & $9.0 / 6.77$ & AT4g00260 & 1 & 4.49 & A. thaliana \\
\hline D11 & CAB80829.1 & $40.9 / 47.9$ & $8.41 / 6.94$ & AT4g04640 & 1 & 3.49 & A. thaliana \\
\hline D12 & NP_904201.1 & 19.6/21.7 & $4.97 / 4.97$ & Photosystem assembly protein ycf3 & 6 & 65.48 & P. patens \\
\hline D13 & BAA83481.1 & $23.3 / 18.4$ & $8.74 / 5.24$ & Rubisco small subunit & 10 & 49.53 & P. patens \\
\hline U1 & CAA11281.1 & $93.9 / 116.8$ & $6.48 / 4.91$ & Stelar $\mathrm{K}^{+}$outward rectifying channel & 1 & 1.45 & A. thaliana \\
\hline U2 & F96615 & $90.8 / 116.8$ & $9.75 / 4.94$ & Probable Myb-family transcription fator & 1 & 2.06 & A. thaliana \\
\hline U3 & AAQ88112.1 & $41.7 / 100.5$ & $5.31 / 5.28$ & Actin & 9 & 37.83 & P. patens \\
\hline U4 & CAB80488.1 & $54.3 / 100.5$ & $5.59 / 5.34$ & Calcium-dependent protein kinase-like protein & 1 & 2.07 & A. thaliana \\
\hline U5 & CAC03450.1 & $44.1 / 96.3$ & $5.78 / 5.27$ & Ser/thr specific protein kinase-like protein & 1 & 3.09 & A. thaliana \\
\hline U6 & D84501 & $13.21 / 97.2$ & $6.75 / 5.33$ & Hypothetical protein At2g12170 & 1 & 10.43 & A. thaliana \\
\hline U7 & CAB79338.1 & $76.5 / 94.7$ & $5.07 / 4.69$ & HSP 70-like protein & 3 & 5.57 & A. thaliana \\
\hline U8 & CAB79338.1 & $76.5 / 83.0$ & $5.07 / 4.80$ & HSP 70-like protein & 3 & 5.57 & A. thaliana \\
\hline U9 & P22954 & 71.4/79.7 & $5.03 / 4.98$ & Heat shock cognate $70 \mathrm{kDa}$ protein 2 & 12 & 24.81 & A. thaliana \\
\hline U10 & CAA05547.1 & 71.1/79.7 & $5.14 / 5.15$ & Heat shock protein 70 & 10 & 12.92 & A. thaliana \\
\hline U11 & CAA05547.1 & $71.1 / 78.8$ & $5.14 / 5.21$ & Heat shock protein 70 & 11 & 19.69 & A. thaliana \\
\hline U12 & CAB87667.1 & $82.1 / 74.8$ & $6.21 / 5.17$ & Subtilisin-like protease-like protein & 1 & 1.85 & A. thaliana \\
\hline U13 & AAM28648.1 & $56.6 / 76.8$ & $4.85 / 4.68$ & Protein disulfide isomerase-like protein & 11 & 27.15 & P. patens \\
\hline U14 & CAB80574.1 & $96.5 / 75.1$ & $5.76 / 4.76$ & Putative receptor-like protein kinase & 1 & 1.59 & A. thaliana \\
\hline U15 & CAB89378.1 & $14.67 / 55.9$ & $7.78 / 4.47$ & Putative proline-rich protein & 1 & 16.92 & A. thaliana \\
\hline U16 & CAB43694.1 & $33.7 / 55.0$ & $10.08 / 4.55$ & Fibrillarin-like protein & 1 & 6.25 & A. thaliana \\
\hline U17 & BAD94943.1 & $40.1 / 48.1$ & $5.13 / 4.41$ & AMP deaminase like protein & 1 & 4.38 & A. thaliana \\
\hline U18 & CAB77733.1 & 27.7/49.7 & $9.58 / 5.06$ & Putative expansin & 1 & 3.53 & A. thaliana \\
\hline U19 & CAB80540.1 & $49.1 / 48.3$ & $9.71 / 5.02$ & Extensin-like protein & 1 & 3.35 & A. thaliana \\
\hline U20 & CAB54558.1 & $47.5 / 46.1$ & $6.04 / 4.88$ & Plastid division protein ftsZ1 & 4 & 18.78 & P. patens \\
\hline U21 & AAY78603.1 & $37.6 / 44.5$ & $5.51 / 4.87$ & PfkB-type carbohydrate kinase family protein & 1 & 3.77 & A. thaliana \\
\hline U22 & Q9FH83 & $156.1 / 44.2$ & $5.61 / 4.80$ & Probable WRKY transcription factor 52 & 1 & 4.46 & A. thaliana \\
\hline U23 & CAB75445.1 & $35.0 / 44.2$ & $5.21 / 4.75$ & Fructokinase-like protein & 1 & 3.99 & A. thaliana \\
\hline U24 & AAG61085.1 & $31.1 / 41.8$ & $5.08 / 4.58$ & Intracellular pathogenesis-related protein-like protein & 1 & 5.17 & P. patens \\
\hline U25 & BAD94888.1 & $44.5 / 38.5$ & $5.09 / 4.98$ & Dnak-type molecular chaperone hsc 70.1-like & 2 & 9.41 & A. thaliana \\
\hline U26 & CAB88528.1 & $33.4 / 38.5$ & 5.245 .09 & bZIP transcription factor-like protein & 1 & 4.39 & A. thaliana \\
\hline U27 & CAC85344.1 & $38.9 / 40.6$ & $8.68 / 5.26$ & Cullin $3 a$ & 1 & 5.33 & A. thaliana \\
\hline U28 & Q39044 & $53.8 / 35.4$ & $5.84 / 4.93$ & Vacuolar processing enzyme, beta-isozyme precursor & 1 & 3.09 & A. thaliana \\
\hline U29 & Q9LQ54 & $80.5 / 36.7$ & $8.87 / 5.38$ & Probable disease resistance protein & 1 & 2.73 & A. thaliana \\
\hline U30 & G96756 & $27.9 / 35.0$ & $5.23 / 5.32$ & Lipoxygenase & 2 & 3.74 & A. thaliana \\
\hline U31 & CAC43712.1 & $34.0 / 33.0$ & $5.9 / 4.80$ & Unnamed protein product & 5 & 17.43 & P. patens \\
\hline U32 & CAC43713.1 & $31.9 / 28.5$ & $5.36 / 4.51$ & Unnamed protein product & 4 & 17.38 & P. patens \\
\hline U33 & CAA67427.1 & $24.5 / 28.5$ & $5.51 / 4.60$ & Thylakoid-bound ascorbate peroxidase & 1 & 3.60 & A. thaliana \\
\hline U34 & F96795 & $135.1 / 30.8$ & $5.5 / 4.75$ & Hypothetical protein F28016.9 & 1 & 1.31 & A. thaliana \\
\hline U35 & Q9LKR3 & $73.6 / 30.4$ & $5.08 / 5.14$ & Luminal binding protein precursor (BiP1) (AtBP1) & 2 & $2 . .54$ & A. thaliana \\
\hline U36 & CAB43552.1 & $36.0 / 30.2$ & $7.08 / 5.28$ & Phosphoribosyl diphosphate synthase & 1 & 6.13 & A. thaliana \\
\hline U37 & CAA66484.2 & $29.1 / 26.8$ & $7.74 / 4.59$ & 2-Cys peroxiredoxin & 1 & 6.02 & A. thaliana \\
\hline U38 & AAV65396.1 & 19.9/23.9 & $5.3 / 5.01$ & Physcomitrin & 4 & 23.60 & P. patens \\
\hline
\end{tabular}


Table 1 Identification of ABA-responsive proteins in P. patens (Continued)

\begin{tabular}{llllllll}
\hline U39 & CAB78663.1 & $26.4 / 22.8$ & $7.77 / 4.89$ & Enoyl-COA hydratase like protein & 1 & 5.74 & A. thaliana \\
U40 & H96806 & $9.9 / 20.6$ & $9.22 / 4.63$ & Unknown protein T32E8.4 & 1 & 19.35 & A. thaliana \\
U41 & AAV65396.1 & $19.9 / 24.1$ & $5.3 / 5.06$ & Physcomitrin & 6 & 38.76 & P. patens \\
U42 & CAB62598.1 & $50.4 / 59.9$ & $6.36 / 6.88$ & N-hydroxycinnamoyl/benzoyltransferase-like protein & 1 & 3.08 & A. thaliana \\
U43 & CAA66816.1 & $42.5 / 49.7$ & $7.62 / 6.08$ & Glyceraldehyde-3-phosphate dehydrogenase & 2 & 7.32 & A. thaliana \\
U44 & CAB87759.1 & $43.9 / 46.3$ & $8.54 / 6.08$ & mRNA binding protein precursor-like & 2 & 5.17 & A. thaliana \\
U45 & CAD38154.1 & $27.7 / 38.4$ & $5.66 / 5.71$ & Putative ascorbate peroxidase & 2 & 13.20 & P. patens \\
U46 & CAD38154.1 & $27.7 / 36.4$ & $5.66 / 5.70$ & Putative ascorbate peroxidase & 3 & 18.80 & P. patens \\
U47 & C96608 & $60.8 / 36.3$ & $8.22 / 5.62$ & Hypothetical protein F25P12.91 & 1 & 3.83 & A.thaliana \\
U48 & CAl84534.1 & $116.4 / 36.1$ & $6.84 / 5.99$ & Unnamed protein product & 1 & 1.35 & A.thaliana \\
U49 & CAA73616.1 & $7.8 / 32.4$ & $9.15 / 5.93$ & Multicatalytic endopeptidase & 2 & 14.29 & A.thaliana \\
U50 & O04005 & $24.1 / 32.3$ & $6.13 / 5.82$ & Peroxiredoxin (Thioredoxin peroxidase) (Rehydrin homolog) & 1 & 6.02 & A.thaliana \\
U51 & CAB43552.1 & $36.0 / 41.9$ & $7.08 / 6.46$ & Phosphoribosyl diphosphate synthase & 1 & 6.13 & A. thaliana \\
U52 & O65719 & $71.1 / 28.4$ & $4.97 / 6.69$ & Heat shock cognate 70 kDa protein 3 & 2 & 4.78 & A.thaliana \\
\hline
\end{tabular}

D1-D13 are down-regulated proteins; U1-U52 are up-regulated, including induced proteins U22, U24, U40,U43; NMP, number of matched peptides; SC, sequence coverage

proteins [28]. Phosphorylation by protein kinases is one of the most common and important regulatory mechanisms in signal transduction among all organisms [29]. These results suggested that these same proteins are involved in the ABA signal transduction pathways of $P$. patens, as reported in higher plants $[6,7]$.

ABA activates the expression of transcriptional regulators Transcriptional control is a major mechanism whereby cells regulate gene expression. Sequence-specific DNAbinding transcriptional regulators, one class of transcription factors, play an essential role in modulating the rate of transcription of specific target genes [30]. The bZIP transcription factors (spot U26) have been shown to regulate diverse biological processes such as pathogen defense, light and stress signaling, seed maturation and flower development [31]. A group of bZIP proteins have been identified as ABRE-binding factors that activate transcription through this cis element [32]. Members of another large family of plant transcription factors, the MYB proteins (spot U2), have also been linked to plant stress responses, including responses to UV light, wounding, anaerobic stress and pathogens [33,34]. WRKY proteins (spot U22) are a novel family of transcription factors that are unique to plants [35]. The WRKY family contains one or two highly conserved WRKY domains characterized by the hallmark heptapeptide WRKYGOK and a zinc-finger structure distinct from other known zinc-finger motifs. Specific WRKY family members show enhanced expression and/or DNA-binding activity following induction by a range of pathogens, defense signals and wounding. These transcriptional regulators have been shown to induce stress-responsive gene expression and protein synthesis and increase plant tolerance to biotic and abiotic stresses in higher plants [36]. Our results suggested that bryophytes, among the earliest land plants, possessed mechanisms of ABA sensing and signal transduction required for stress responses, similar to higher plants.
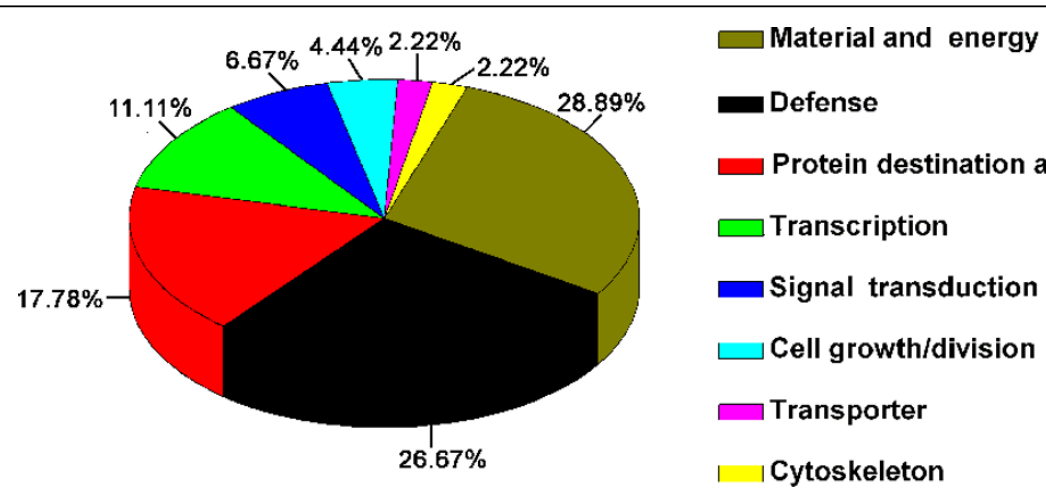

Figure 3 Functional categorization of the up- and down-regulated $P$. patens proteins in response to ABA treatment. Numerical values represent the percentage of proteins in each functional category. 
Table 2 Classes of proteins identified on two-dimensional gels

\begin{tabular}{|c|c|}
\hline Protein Category & Spot no. \\
\hline \multicolumn{2}{|l|}{ Material and energy metabolism (28.89\%) } \\
\hline ATP synthase beta subunit & D3 $\downarrow^{a}$ \\
\hline $\begin{array}{l}\text { Putative protein } 1 \text { photosystem II oxygen-evolving } \\
\text { complex }\end{array}$ & D4 $\downarrow$ \\
\hline Rubisco small subunit & D9 $\downarrow, \mathrm{D} 13 \downarrow$ \\
\hline Photosystem assembly protein ycf3 & D12 $\downarrow$ \\
\hline AMP deaminase like protein & $\mathrm{U} 17 \uparrow^{\mathrm{b}}$ \\
\hline PfkB-type carbohydrate kinase family protein & U21 \\
\hline Fructokinase-like protein & U23 $\uparrow$ \\
\hline Enoyl-COA hydratase like protein & U39 $\uparrow$ \\
\hline Glyceraldehyde-3-phosphate dehydrogenase & U $43 * c$ \\
\hline Multicatalytic endopeptidase & U49 ^ \\
\hline Phosphoribosyl diphosphate synthase & U36 $\uparrow, U 51 \uparrow$ \\
\hline Subtilisin-like protease-like protein & $\mathrm{U} 12 \uparrow$ \\
\hline Protein disulfide isomerase-like protein & U13 $\uparrow$ \\
\hline \multicolumn{2}{|l|}{ Defense $(26.67 \%)$} \\
\hline Putative proline-rich protein & U15 $\uparrow$ \\
\hline Putative expansin & U18 $\uparrow$ \\
\hline Extensin-like protein & U19 \\
\hline Intracellular pathogenesis-related protein-like protein & U24 * \\
\hline Disease resistance protein RPM1 & U29 ^ \\
\hline Lipoxygenase & U30 个 \\
\hline Thylakoid-bound ascorbate peroxidase & U33 $\uparrow$ \\
\hline 2-Cys peroxiredoxin & U37 $\uparrow$ \\
\hline Physcomitrin & 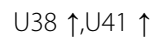 \\
\hline N-hydroxycinnamoyl/benzoyltransferase-like protein & U42 \\
\hline Putative ascorbate peroxidase & 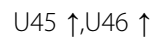 \\
\hline Peroxiredoxin & U50个 \\
\hline \multicolumn{2}{|l|}{ Protein destination and storage (17.78\%) } \\
\hline Chloroplast FtsH protease & D1 $\downarrow$ \\
\hline HSP 70-like protein & 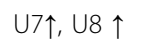 \\
\hline Heat shock cognate $70 \mathrm{kDa}$ protein 2 & U9 $\uparrow$ \\
\hline Heat shock protein 70 & $\begin{array}{l}\text { U10 } \uparrow, ~ U 11 \\
\uparrow\end{array}$ \\
\hline DnaK-type molecular chaperone hsc 70.1-like & U25 $\uparrow$ \\
\hline Cullin 3a & U27 $\uparrow$ \\
\hline Luminal binding protein precursor (BiP1) (AtBP1) & U35 $\uparrow$ \\
\hline Heat shock cognate $70 \mathrm{kDa}$ protein 3 & U52 $\uparrow$ \\
\hline \multicolumn{2}{|l|}{ Transcription (11.11\%) } \\
\hline Probable Myb-family transcription fator & $\mathrm{U} 2 \uparrow$ \\
\hline Fibrillarin-like protein & U16 \\
\hline Probable WRKY transcription factor 52 & $\mathrm{U} 22$ * \\
\hline bZIP transcription factor-like protein & U26 $\uparrow$ \\
\hline mRNA binding protein precursor-like & 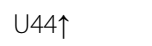 \\
\hline \multicolumn{2}{|l|}{ Signal transduction $(6.67 \%)$} \\
\hline Calcium-dependent protein kinase-like protein & U4 $\uparrow$ \\
\hline Ser/thr specific protein kinase-like protein & U5 $\uparrow$ \\
\hline Putative receptor-like protein kinase & U14 $\uparrow$ \\
\hline \multicolumn{2}{|l|}{ Cell growth/division (4.44\%) } \\
\hline Plastid division protein ftsZ1 & U20个 \\
\hline Vacuolar processing enzyme, beta-isozyme precursor & U28个 \\
\hline
\end{tabular}

Table 2 Classes of proteins identified on two-dimensional gels (Continued)

Transporter (2.22\%)

Stelar $\mathrm{K}^{+}$outward rectifying channel U1

Cytoskeleton (2.22\%)

Actin U3१

a The downward arrow represents the down-regulated protein spot on the 2-D gel;

${ }^{b}$ The upward arrow represents the up-regulated protein spot on the 2-D gel;

${ }^{C}$ The * represents the newly induced protein spot on the 2-D gel.

$A B A$ induces molecular chaperone synthesis

It is well known that Hsps are induced by ABA in higher plants. Hsps/chaperones can play a crucial role in protecting plants against stress by reestablishing normal protein conformation and thus cellular homeostasis. Five major families of Hsps are conservatively recognized: the Hsp70 (DnaK) family; the chaperonins (GroEL and Hsp60); the Hsp90 family; the Hsp100 (Clp) family; and the small Hsp (sHsp) family [37]. The Hsp70 family (spots U7, U8, U9, U10, U11, U25, U35, U52) represents the most highly conserved of the HSPs, with functional counterparts found in the most primitive of bacteria and the most sophisticated of higher organisms [38]. HSP70 s are involved in almost every step of protein biogenesis [39]. They play essential roles as molecular chaperones assisting the correct folding of nascent polypeptide chains as they emerge from the ribosome, participating in transmembrane protein transport, and limiting cellular damage following stress by their ability to prevent protein aggregation and to restore the function of denatured proteins $[40,41]$. Luminal binding protein (BiP) (spot U35) is a member of the Hsp70 family that is localized to the endoplasmic reticulum (ER) of eukaryotic cells, where it functions as a chaperone and is believed to support proper protein folding and protein translocation into the ER lumen [42]. HSP is a large protein family that includes HSP90 and HSP60, among others. In our study, different members of Hsp70 subfamily were the major HSPs that were up-regulated by ABA treatment in P. patens.

\section{$A B A$ triggers stress and defense responses}

As in higher plants, many defense proteins were upregulated by ABA signal. In this study, we identified twelve proteins related to defense and cellular protection. Intracellular pathogenesis-related proteins (spot U24) are defined as proteins that are induced by pathogens attack as well as abiotic stimuli. They are powerful defense agents that are currently being use to genetically enhance cereals susceptible to endemic disease and insect pests [43]. In order to combat pathogen infection, plants also produce disease resistance protein (spot U29) that specifically detects the appearance of avirulence products. If they lack corresponding disease 
resistance proteins, avirulence products can function as virulence factors, subverting host cellular functions through interactions with plant-encoded pathogenicity targets [44]. Lipoxygenases (LOXs) (spot U30) catalyze the formation of fatty acid hydroperoxides involved in response to stresses such as pathogen attack, wounding, water deficit, and anoxia [45]. N-hydroxycinnamoyl/benzoyl transferase (HCBT) (spot U42) catalyses a reaction in the phytoalexin biosynthetic pathway in carnation [46]. Extensin (spots U18, U19) is a hydroxyproline-rich glycoprotein (HRGP) found in the cell walls of higher plants [47]. It is induced by many stimuli such as jasmonic acid, wounding and pathogen attack and appears to play an important part in the plant defense response $[48,49]$. Proline-rich proteins (spot U15) are also cell wall proteins that actively contribute to the plant defense response [50]. Enzymes involved in antioxidation include ascorbate peroxidase (spots U33, U45, U46), which catalyses the first reaction between $\mathrm{H}_{2} \mathrm{O}_{2}$ and ascorbate in the ascorbate-glutathione cycle, giving rise to monohydroascorbate and $\mathrm{H}_{2} \mathrm{O}$ [51]; and peroxiredoxin (spots. U37, U50), which reduces $\mathrm{H}_{2} \mathrm{O}_{2}$ and detoxifies alkyl hydroperoxides and peroxinitrite [52]. Physcomitrin (spots U38, U41) is a novel plant cytolysin but its biological function is not clear. It is significantly induced by $\mathrm{ABA}$ treatment, $\mathrm{NaCl}$, and desiccation $[53,54]$. and is required Further study is required to determine whether physcomitrin makes a significant contribution in response to extreme environmental conditions in $P$. patens. The identification of a large number of defense proteins implies that ABA could increase the resistance of $P$. patens towards pathogens and abiotic stresses, protecting the plant from oxidative damage.

\section{ABA inhibits photosynthesis in P. Patens}

Proteins involved in photosynthesis and carbon assimilation that responded to ABA treatment are represented by the small subunit of Rubisco (spots D9, D13); photosystem II oxygen-evolving complex proteins (spot D4), that catalyze acid-base reactions or that are involved in proton uptake and release [55]; photosystem assembly protein ycf3 (spot D12), which regulates the assembly of photosystem complex proteins. Down-regulation of these proteins suggests that photosynthesis may be inhibited by ABA in $P$. patens. These results were in accord with previous data which showed ABA inhibits photosynthesis in higher plant [8]. Together, our results indicated that ABA-responsive bryophytes underwent physiological changes that had features similar to those of higher plants.

\section{Conclusions}

Mosses were one of the earliest land plants in evolutionary terms, and have many characteristics in common with seed plants. Therefore, a process common to land plants, such as responding to the plant phytohormone ABA, can be worthwhile to study in the moss species, $P$. patens. In order to investigate global protein changes after treatment with ABA, we have performed a comparative proteome analysis of $P$. patens and revealed a complex regulatory network. Upon the application of ABA, $P$. patens cells can sense ABA and transmit a signal, which, in turn, activates transcriptional factormediated metabolic and defensive genes expression. In higher plants, a broad spectrum of genes is also induced by the ABA treatment, and these genes encode not only proteins involved in the protection of cellular structures or in the biosynthesis of protective metabolites but also key regulatory proteins that induce stress-responsive gene expression and protein synthesis and increase plant tolerance to biotic and abiotic stresses. These responses are similar to those seen in the moss $P$. patens in response to ABA. Figure 4 illustrates the ABA response process of angiosperm (A. thaliana; from reference 6,7 ) and bryophyte ( $P$. patens). Based on these results, it seems likely that $P$. patens and higher plants share common receptor protein, protein kinases, transcription factors and physiological and biochemical reactions.

\section{Methods}

\section{Plant materials}

$P$. patens was grown in modified BCD medium containing $0.5 \%(\mathrm{w} / \mathrm{v})$ glucose and $0.75 \%(\mathrm{w} / \mathrm{v})$ agar [56]. Gametophores were cultured under the standard conditions $\left(23^{\circ} \mathrm{C}\right)$ with a light cycle of $16 \mathrm{~h}$ light $/ 8 \mathrm{~h}$ darkness and a light intensity of $55 \mu \mathrm{mol} \mathrm{s} \mathrm{m}^{-1}$. Three-week-old gametophores were treated with $0 \mu \mathrm{M}$ or $50 \mu \mathrm{M}$ ABA for $72 \mathrm{~h}$. For ABA treatment, gametophores were transferred to BCD solid medium supplemented with $50 \mu \mathrm{M}$ $( \pm)$-cis-trans ABA. This treatment was carried out three separate times [57]. Gametophores were harvested, frozen in liquid nitrogen and stored at $-80^{\circ} \mathrm{C}$.

\section{Chemicals}

IPG Drystrip, IPG buffer (pH 3-10), TEMED, ammonium persulfate, acrylamide, SDS and Bis were purchased from GE Healthcare BIO-Science (Uppsala, Sweden); CHAPS, urea, thiourea, iodoacetamide, ammonium bicarbonate, trypsin were purchased from Sigma (St. Louis, MO, USA); DTT and glycine were purchased from Amresco (Solon, OH, USA); ZipTipC $18 \mathrm{P}^{\mathrm{TM}}$ pipette tips were purchased from Millipore (Bedford, MA, USA).

\section{Protein extraction and 2-DE}

Proteins were extracted using a phenol extraction procedure [54]. The resulting pellets were dissolved in a sample buffer (7 M urea, $2 \mathrm{M}$ thiourea, 4\% (w/v) CHAPS, 


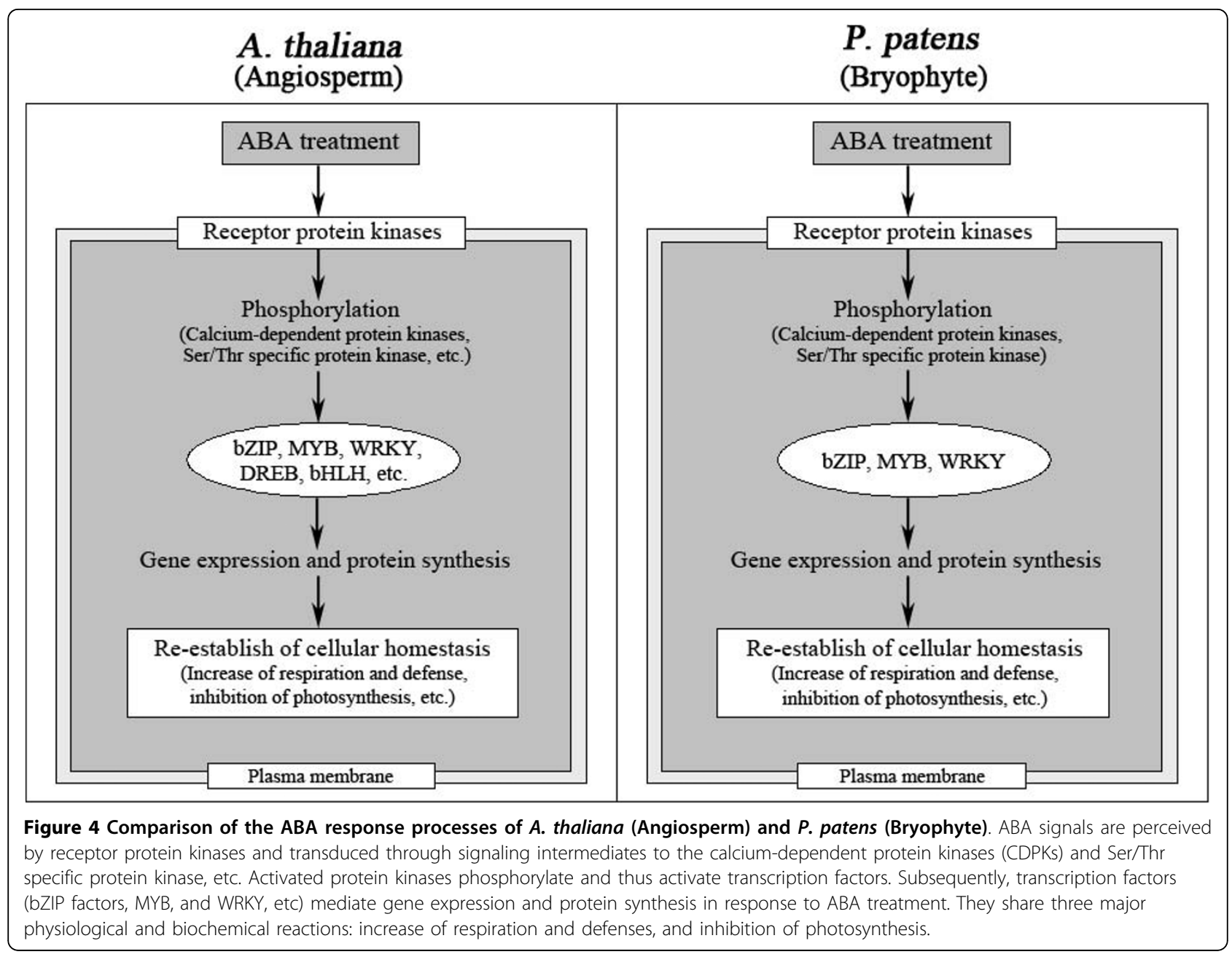

$0.5 \%(\mathrm{v} / \mathrm{v})$ IPG buffer, $1 \%(\mathrm{w} / \mathrm{v})$ DTT) at room temperature. 2-D electrophoresis was carried out according to Bjellquist et al. [58]. Dry IPG strips (13 cm long, pH 4-7 linear) were rehydrated for $12 \mathrm{~h}$ in $250 \mu \mathrm{l}$ rehydration buffer containing $800 \mu \mathrm{g}$ protein samples. Isoelectric focusing was conducted at $20^{\circ} \mathrm{C}$ with an Ettan IPGphor system (GE Healthcare Amersham Bioscience, Little Chalfont, UK). Focusing was performed and focused strips were then equilibrated as described previously [54]. For the second dimension, the proteins were separated on $15 \%$ SDS polyacrylamide gels. Gels were stained with $0.1 \%$ Coomassie brilliant blue (CBB) R-250 in $25 \%$ ethanol $/ 8 \%$ acetic acid and destained in $25 \%$ ethanol $/ 8 \%$ acetic acid.

\section{Image and data analysis}

The 2-DE gels were scanned at 600 dpi with a UMAX Power Look 2100XL scanner (Maxium Tech Inc., Taiwan, China). The transparency mode was used to obtain a grayscale image. The image analysis was performed with ImageMaster $^{\mathrm{mi}} 2 \mathrm{D}$ Platinum software. To verify the auto detected results, all spots were manually inspected and edited as necessary. After spot detection, quantification, and background subtraction, each analyzed gel was matched individually to the reference gel. To compensate for subtle differences in sample loading, gel staining, and destaining, the volume of each spot (i.e. spot abundance) was normalized as a relative volume. Only those with reproducible changes (quantitative changes more than 1.5 -fold in abundance and $t$ - test $\mathrm{P}<0.05$ ) among three replicates were used for further analysis.

\section{In-gel digestion and protein identification}

Protein spots were manually excised from the gel. In-gel digestion by trypsin and LC-MS/MS were performed according to Wang et al. [54]. All MS/MS samples were analyzed using the TurboSEQUEST program in the BioWorks 3.1 software suite (Thermo), against a database adapted from $P$. patens and $A$. thaliana NCBI protein database, in which each genuine protein sequence was followed by a reversed amino acid sequence. The search parameters included, up to two missed trypsin cleavages 
with carboxamidomethylation at cysteine. The mass tolerance for precursor ions and fragment ions were considered as 2 amu and 1 amu respectively. Peptides had to be fully tryptically digested and be at least seven amino acids long in the searched databases. The output results were combined together using the software BuildSummary. False positive rate (FPR) was kept less than $5 \%$ as the filtration criterion. The FPR was calculated as double of the number of peptides from reversed database divided by the number of peptides from reversed and normal database [59]. An accepted SEQUEST result had a $\Delta \mathrm{Cn}$ score of at least 0.1 (regardless of charge state). Peptides with a +1 charge state were accepted if they were fully tryptic digested and had a cross correlation (Xcorr) of at least 1.9. Peptides with a +2 charge state were accepted if they had an Xcorr $\geq 2.2$. Peptides with a +3 charge state were accepted if they had an Xcorr $\geq 3.75$.

\section{Additional material}

Additional file 1: Supplemental Table S1 Quantitative changes in spot intensities. The protein abundance is presented as the percentage of the total spot volume associated with each identified spot. The Vol\% value of all identified proteins and the data of statistical analysis among three replicates are listed in this file.

Additional file 2: Supplemental Table S2 Peptides sequence matched of $A B A$ responsive proteins. This file includes the peptides sequence of all identified proteins, total peptide count and unique peptide count which are searched, and charge, XCorr value and score of every peptide.

\section{Acknowledgements}

This work was supported by grants from National Key Project for Gene Transform in China (2009ZX08009-058B), Beijing National Science Key Foundation (KZ200610028017 and 5021001), Chinese 863 Project (2007AA021405), Institutions of Higher Learning under the Jurisdiction of Beijing Municipality for Academic Human Resources Development to He.

\section{Author details}

${ }^{1}$ College of Life Sciences, Capital Normal University, Beijing 100048, China. ${ }^{2}$ Key Laboratory of Urban Agriculture (North) of Ministry of Agriculture P. R. China, Beijing 102206, China. ${ }^{3}$ Beijing University of Agriculture, Beijing 102206, China. ${ }^{4}$ Department of Biology, Washington University in St. Louis, MO 63130, US.

\section{Authors' contributions}

XQW YKH and TYK Conceived and designed the study. XQW Performed the experiments. XQW Analyzed the data. YKH and XQW Contributed reagents/ materials/analysis tools. XQW Wrote the paper. All authors read and approved the final manuscript.

Received: 26 December 2009 Accepted: 27 August 2010 Published: 27 August 2010

\section{References}

1. Rock CD: Pathways to abscisic acid-regulated gene expression. New Phytol 2000, 148(3):357-396.

2. Hirsch $\mathrm{R}$, Hartung $\mathrm{W}$, Gimmler H: Abscisic acid content in algae under stress. Bot Acta 1989, 102(44):326-334.
3. Yamamoto $H$, Inomata M, Tsuchiya S, Nakamura M, Uchiyama T, Oritani T: Early biosynthetic pathway to abscisic acid in Cercospora cruenta. Biosci Biotechnol Biochem 2000, 64(10):2075-2082.

4. Le Page-Degivry MT, Bidard JN, Rouvier E, Bulard C, Lazdunski M: Presence of abscisic acid, a phytohormone, in the mammalian brain. Proc Natl Acad Sci USA 1986, 83(4):1155-1158.

5. Fedoroff NV: Cross-talk in abscisic acid signaling. Sci STKE 2002, 2002(140): RE10.

6. Seki M, Ishida J, Narusaka M, Fujita M, Nanjo T, Umezawa T, Kamiya A Nakajima M, Enju A, Sakurai T, et al: Monitoring the expression pattern of around 7,000 Arabidopsis genes under ABA treatments using a fulllength cDNA microarray. Funct Integr Genomics 2002, 2(6):282-291.

7. Li Y, Lee KK, Walsh S, Smith C, Hadingham S, Sorefan K, Cawley G, Bevan MW: Establishing glucose- and ABA-regulated transcription networks in Arabidopsis by microarray analysis and promoter classification using a Relevance Vector Machine. Genome Res 2006, 16(3):414-427.

8. Rakwal R, Komatsu S: Abscisic acid promoted changes in the protein profiles of rice seedling by proteome analysis. Mol Biol Rep 2004, 31(4):217-230

9. Cove D, Bezanilla M, Harries P, Quatrano R: Mosses as model systems for the study of metabolism and development. Annu Rev Plant Biol 2006, 57:497-520.

10. Reski R: Physcomitrella and Arabidopsis: the David and Goliath of reverse genetics. Trends in Plant Science 1998, 3(6):209-210.

11. Schumaker KS, Dietrich MA: Hormone-Induced Signaling During Moss Development. Annu Rev Plant Physiol Plant Mol Biol 1998, 49:501-523.

12. Cove DJ, Knight CD, Lamparter T: Mosses as model systems. Trends in Plant Science 1997, 2(3):99-105.

13. Reski R: Development, genetics and molecular biology of mosses. Bot Acta 1998, 111(1):1-15.

14. Mishler BD, Lewis LA, Buchheim MA, Renzaglia KS, Garbarg DJ, Delwiche CF, Zechman FW, Kantz TS, Chapman RL: Phylogenetic relationships of the green "algae" and "bryophytes". Ann Missouri Bot Gard 1994, 81(3):451-483.

15. Oliver MJ, Wood AJ: Desiccation tolerance in mosses. Stress-inducible processes in higher eukaryotic cells Plenum Publishing Corp. New York 1997, $1-26$.

16. Reski R, Reynolds S, Wehe M, Kleber-Janke T, Kruse S: Moss (Physcomitrella patens) expressed sequence tags include several sequences which are novel for plants. Bot Acta 1998, 111(2):143-149.

17. Quatrano RS, McDaniel SF, Khandelwal A, Perroud PF, Cove DJ: Physcomitrella patens: mosses enter the genomic age. Curr Opin Plant Biol 2007, 10(2):182-189.

18. Minami A, Nagao M, Arakawa K, Fujikawa S, Takezawa D: Abscisic acidinduced freezing tolerance in the moss Physcomitrella patens is accompanied by increased expression of stress-related genes. J Plant Physiol 2003, 160(5):475-483.

19. Minami A, Nagao M, Ikegami K, Koshiba T, Arakawa K, Fujikawa S, Takezawa D: Cold acclimation in bryophytes: low-temperature-induced freezing tolerance in Physcomitrella patens is associated with increases in expression levels of stress-related genes but not with increase in level of endogenous abscisic acid. Planta 2005, 220(3):414-423.

20. Oldenhof H, Wolkers WF, Bowman JL, Tablin F, Crowe JH: Freezing and desiccation tolerance in the moss Physcomitrella patens: an in situ Fourier transform infrared spectroscopic study. Biochim Biophys Acta 2006, 1760(8):1226-1234

21. Nagao M, Minami A, Arakawa K, Fujikawa S, Takezawa D: Rapid degradation of starch in chloroplasts and concomitant accumulation of soluble sugars associated with ABA-induced freezing tolerance in the moss Physcomitrella patens. J Plant Physio/ 2005, 162(2):169-180.

22. Nishiyama T, Fujita T, Shin IT, Seki M, Nishide H, Uchiyama I, Kamiya A, Carninci P, Hayashizaki Y, Shinozaki K, et al: Comparative genomics of Physcomitrella patens gametophytic transcriptome and Arabidopsis thaliana: implication for land plant evolution. Proc Natl Acad Sci USA 2003, 100(13):8007-8012.

23. Bevan M, Bancroft I, Bent E, Love K, Goodman H, Dean C, Bergkamp R, Dirkse W, Van Staveren M, Stiekema W, et al: Analysis of $1.9 \mathrm{Mb}$ of contiguous sequence from chromosome 4 of Arabidopsis thaliana. Nature 1998, 391(6666):485-488 
24. Fantl WJ, Johnson DE, Williams LT: Signaling by receptor tyrosine kinases. Annu Rev Biochem 1993, 62:453-481.

25. Braun DM, Walker JC: Plant transmembrane receptors: new pieces in the signaling puzzle. Trends Biochem Sci 1996, 21(2):70-73.

26. Hong SW, Jon JH, Kwak JM, Nam HG: Identification of a receptor-like protein kinase gene rapidly induced by abscisic acid, dehydration, high salt, and cold treatments in Arabidopsis thaliana. Plant Physiol 1997, 113(4):1203-1212.

27. Ludwig AA, Romeis T, Jones JD: CDPK-mediated signalling pathways: specificity and cross-talk. J Exp Bot 2004, 55(395):181-188.

28. Rudrabhatla P, Reddy MM, Rajasekharan R: Genome-wide analysis and experimentation of plant serine/threonine/tyrosine-specific protein kinases. Plant Molecular Biology 2006, 60(2):293-319.

29. Hardie DG: PLANT PROTEIN SERINE/THREONINE KINASES: Classification and Functions. Annu Rev Plant Physiol Plant Mol Biol 1999, 50:97-131.

30. Martinez E: Multi-protein complexes in eukaryotic gene transcription. Plant Mol Biol 2002, 50(6):925-947.

31. Jakoby M, Weisshaar B, Droge-Laser W, Vicente-Carbajosa J, Tiedemann J, Kroj T, Parcy F: bZIP transcription factors in Arabidopsis. Trends Plant Sci 2002, 7(3):106-111

32. Kobayashi Y, Murata M, Minami H, Yamamoto S, Kagaya Y, Hobo T, Yamamoto A, Hattori T: Abscisic acid-activated SNRK2 protein kinases function in the gene-regulation pathway of $A B A$ signal transduction by phosphorylating ABA response element-binding factors. Plant J 2005, 44(6):939-949.

33. Rushton PJ, Somssich IE: Transcriptional control of plant genes responsive to pathogens. Curr Opin Plant Biol 1998, 1(4):311-315.

34. Stracke R, Werber M, Weisshaar B: The R2R3-MYB gene family in Arabidopsis thaliana. Current Opinion in Plant Biology 2001, 4(5):447-456.

35. Eulgem T, Rushton PJ, Robatzek S, Somssich IE: The WRKY superfamily of plant transcription factors. Trends Plant Sci 2000, 5(5):199-206.

36. Singh K, Foley RC, Onate-Sanchez L: Transcription factors in plant defense and stress responses. Curr Opin Plant Biol 2002, 5(5):430-436.

37. Wang W, Vinocur B, Shoseyov O, Altman A: Role of plant heat-shock proteins and molecular chaperones in the abiotic stress response. Trends Plant Sci 2004, 9(5):244-252.

38. Boorstein WR, Ziegelhoffer T, Craig EA: Molecular evolution of the HSP70 multigene family. J Mol Evol 1994, 38(1):1-17.

39. Hartl FU: Molecular chaperones in cellular protein folding. Nature 1996, 381(6583):571-579

40. Clarke AR: Molecular chaperones in protein folding and translocation. Curr Opin Struct Biol 1996, 6(1):43-50.

41. Parsell DA, Lindquist $\mathrm{S}$ : The function of heat-shock proteins in stress tolerance: degradation and reactivation of damaged proteins. Annu Rev Genet 1993, 27:437-496.

42. Gething MJ, Sambrook J: Protein folding in the cell. Nature 1992, 355(6355):33-45.

43. Muthukrishnan S, Liang GH, Trick HN, Gill BS: Pathogenesis-related proteins and their genes in cereals. Plant Cell Tiss Org 2001, 64(23):93-114.

44. van der Biezen EA, Jones JDG: Plant disease-resistance proteins and the gene-for-gene concept. Trends in Biochemical Sciences 1998, 23(12):454-456.

45. Porta H, Rocha-Sosa M: Plant lipoxygenases. Physiological and molecular features. Plant Physiol 2002, 130(1):15-21.

46. Yang Q, Grimmig B, Matern U: Anthranilate N-hydroxycinnamoyl/ benzoyltransferase gene from carnation: rapid elicitation of transcription and promoter analysis. Plant Mol Biol 1998, 38(6):1201-1214.

47. Kieliszewski MJ, Lamport DTA: Extensin repetitive motifs functional sites posttranslational codes and phylogeny. Plant J 1994, 5(2):157-172.

48. Creelman RA, Tierney ML, Mullet JE: Jasmonic acid/methyl jasmonate acumulate in wounded soybean hypocotyls and modulate wound gene expression. Proc Natl Acad Sci USA 1992, 89(11):4938-4941.

49. Kawalleck P, Schmelzer E, Hahlbrock K, Somssich IE: Two pathogen responsive genes in parsley encode a tyrosinerich hydroxyprolinerich glycoprotein (hrgp) and an anionic peroxidase. Mol Gen Genet 1995, 247(4):444-452.

50. Bradley DJ, Kjellbom P, Lamb CJ: Elicitor- and wound-induced oxidative cross-linking of a proline-rich plant cell wall protein: a novel, rapid defense response. Cell 1992, 70(1):21-30.
51. D'Arcy-Lameta A, Ferrari-lliou R, Contour-Ansel D, Pham-Thi AT, ZuilyFodil Y: Isolation and characterization of four ascorbate peroxidase cDNAs responsive to water deficit in cowpea leaves. Ann Bot (Lond) 2006, 97(1):133-140

52. Dietz KJ, Jacob S, Oelze ML, Laxa M, Tognetti V, de Miranda SM, Baier M, Finkemeier I: The function of peroxiredoxins in plant organelle redox metabolism. J Exp Bot 2006, 57(8):1697-1709.

53. Wang XQ, Yang PF, Gao Q, Liu XL, Kuang TY, Shen SH, He YK: Proteomic analysis of the response to high-salinity stress in Physcomitrella patens. Planta 2008, 228:167-177.

54. Wang XQ, Yang PF, Liu Z, Liu WZ, Hu Y, Chen H, Kuang TY, Pei ZM, Shen SH, He YK: Exploring the mechanism of Physcomitrella patens desiccation tolerance through a proteomic strategy. Plant Physiol 2009, 149(4):1739-1750

55. Coleman WJ: Chloride binding proteins: mechanistic implications for the oxygen-evolving complex of Photosystem II. Photosynthesis Reseach 1990, 23(1):1-27.

56. Ashton NW, Cove DJ, Featherstone DR: The isolation and physiological analysis of mutants of the moss Physcomitrella patens, which overproduce gametophores. Planta 1979, 144(5):437-442.

57. Frank W, Ratnadewi D, Reski R: Physcomitrella patens is highly tolerant against drought, salt and osmotic stress. Planta 2005, 220(3):384-394

58. Bjellqvist B, Ek K, Righetti PG, Gianazza E, Görg A, Westermeier R, Postel W: Isoelectric focusing in immobilized $\mathrm{pH}$ gradients: principle, methodology and some applications. Journal of Biochemical and Biophysical Methods 1982, 6(4):317-339

59. Ning ZB, Li QR, Dai J, Li RX, Shieh CH, Zeng R: Fractionation of Complex Protein Mixture by Virtual Three-Dimensional Liquid Chromatography Based on Combined pH and Salt Steps. Journal of Proteome Reseach 2008, 7:4525-4537.

doi:10.1186/1471-2229-10-192

Cite this article as: Wang et al:: Conservation between higher plants and the moss Physcomitrella patens in response to the phytohormone abscisic acid: a proteomics analysis. BMC Plant Biology 2010 10:192.

\section{Submit your next manuscript to BioMed Central and take full advantage of:}

- Convenient online submission

- Thorough peer review

- No space constraints or color figure charges

- Immediate publication on acceptance

- Inclusion in PubMed, CAS, Scopus and Google Scholar

- Research which is freely available for redistribution 